Guido Dette

\title{
Kursbildung \\ am deutschen \\ Aktienmarkt
}

Mit einem Geleitwort von

Prof. Dr. Wolfgang Benkert

Springer Fachmedien Wiesbaden $\mathrm{GmbH}$ 


\section{Dette, Guido:}

Kursbildung am deutschen Aktienmarkt / Guido Dette. Mit einem Geleitw. von Wolfgang Benkert. - Wiesbaden: Dt. Univ.Verl., 1998

(DUV: Wirtschaftswissenschaft)

Zugl.: Witten, Herdecke, Privatuniv., Diss., 1997

ISBN 978-3-8244-0382-0

Alle Rechte vorbehalten

(C) Springer Fachmedien Wiesbaden, 1998

Ursprünglich erschienin bei Deutscher Universitäts-Verlag GmbH, Wiesbaden, 1998

Lektorat: Monika Mülhausen

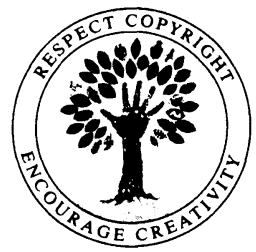

Das Werk einschließlich aller seiner Teile ist urheberrechtlich geschützt. Jede Verwertung außerhalb der engen Grenzen des Urheberrechtsgesetzes ist ohne Zustimmung des Verlages unzulässig und strafbar. Das gilt insbesondere für Vervielfältigungen, Übersetzungen, Mikroverfilmungen und die Einspeicherung und Verarbeitung in elektronischen Systemen.

Gedruckt auf säurefreiem Papier

ISBN 978-3-8244-0382-0

ISBN 978-3-663-08686-4 (eBook)

DOI 10.1007/978-3-663-08686-4 


\section{Geleitwort}

Zwischen Börsenpraxis und der wissenschaftlichen Beschäftigung mit dem Börsengeschehen scheinen Welten zu liegen: Die Börsenpraxis folgt nach dem Eindruck distanzierter Beobachter Stimmungen, self-fulfilling prophecies, wenn sie nicht gar von Massenpsychosen bestimmt wird. Die Wissenschaft - so der Eindruck vieler Börsenpraktiker - hingegen verfeinert ständig hochkomplexe Modelle, ohne nach den fundamentalen Bedingungen von Börsentrends zu fragen.

Die vorliegende Arbeit, die auf einer der wirtschaftswissenschaftlichen Fakultät der Universität Witten/Herdecke vorgelegten Dissertation basiert, versucht eine Brücke zwischen der scheinbar theoriefernen Börsenpraxis und der praxisfernen Wissenschaft zu schlagen: Der Autor - selbst im Portfolio-Management tätig - kennt die Mechanismen der Börse und kann seine Thesen an vielen Börsenerfahrungen belegen. Er analysiert das Börsengeschehen im Lichte eines Theorieansatzes, der verhaltensökonomischen Forschung, dessen Vertreter selbst einen hohen Anspruch an deren Anwendbarkeit und Umsetzbarkeit stellen. Angewandt auf das Börsengeschehen gelingt es dem Autor, eine Vielzahl fundamentaler und meßbarer Einflußgrößen zu identifizieren, die vor allem in langfristiger Perspektive durch Einbeziehung des wirtschaftlichen, gesellschaftlichen und kulturellen Umfeldes, in dem Anlageentscheidungen getroffen werden, diese Entscheidungen und damit die Entwicklung von einzelnen Aktien und ganzen Börsen erklären helfen.

Die Entwicklung eines erklärungsmächtigen Theorieansatzes vor dem Hintergrund börsenpraktischer Erfahrungen macht das vorliegende Buch für theoretisch Interessierte wie für Praktiker gleichermaßen lesenswert. Das Buch ist hilfreich für das Verstehen des Börsengeschehens - eine Herausforderung für die Wissenschaft wie für die Praxis.

Prof. Dr. Wolfgang Benkert 


\section{Vorwort}

Jeden Börsianer beschäftigt die Frage, ob es an der Börse Strategien gibt, die einen überdurchschnittlichen Erfolg ermöglichen. Es überrascht deshalb nicht, daß es eine Vielzahl von Publikationen über Aktienkursprognosen gibt. Die meisten von ihnen unterstellen, daß generell das Verhalten der Marktteilnehmer faktisch unbedeutend sei und daß die Verhaltensweisen der Einzelnen, falls überhaupt, nur auf der Grundlage idealisierter Informations- und Verhaltensannahmen Untersuchungsgegenstand sein könnten. Die in der praktischen Tätigkeit als Analyst und Portfolio Manager gewonnenen Erfahrungen machten die Brüchigkeit dieser Annahme aber zunehmend deutlich. Die vorliegende Arbeit folgt ihr deshalb nicht; sie berücksichtigt vielmehr verstärkt die einzelwirtschaftliche Ebene und stellt den Informationsverarbeitungsprozeß und das Verhalten einzelner Marktteilnehmer in den Vordergrund, wie es im täglichen Börsengeschehen zu beobachten ist und zieht hieraus Schlüsse auf die Aktienkursentwicklung. Den komplexen Entscheidungsprozeß sowie das individuelle Verhalten gilt es einzugrenzen, $\mathrm{zu}$ analysieren und letztlich in die Aktienanalyse $\mathrm{zu}$ integrieren. Welche Wege hierfür zur Verfügung stehen und inwieweit sie praktikabel sind, wird in dem vorliegenden Buch detailliert beschrieben. Es wird nicht ein vielversprechendes System oder eine neue Handelstechnik in den Mittelpunkt gestellt, sondern statt dessen der Aktieninvestor selbst, der der wichtigste Faktor der Aktienkursbildung ist und dadurch wird eine praktikable und vor allem höhere Prognosegüte ermöglicht.

Am Entstehen dieser Arbeit haben im Rückblick viele Personen in verschiedenster Weise, bspw. in Diskussionen und zum Nachdenken Anlaß gebende Anregungen, Hilfestellung geleistet. Nicht alle kann ich deshalb namentlich benennen und Ihnen danken.

Meinen aufrichtigen und herzlichen Dank möchte ich meinem Doktorvater, Herrn Professor Dr. Wolfgang Benkert, für die so wohlwollende Unterstützung dieser Arbeit und für seine wertvollen Ratschläge aussprechen.

Zu Dank verpflichtet bin ich meinem vorherigen Arbeitgeber, der Oppenheim Finanzanalyse, der mir während meiner Promotion durch eine flexible Arbeitszeit entgegenkam und dem 
Gerling Konzern, der mir eine selbständige Arbeit mit großer Verantwortungsübertragung ermöglichte, durch die ich erst tief reichende und detaillierte Einblicke in das tägliche Börsengeschehen erhielt, die mir bei einer ausschließlich theoretischen Bearbeitung verschlossen geblieben wären.

Ganz besonderen Dank schulde ich meinen Eltern, denen ich diese Arbeit widmen möchte.

Guido Dette 


\section{Inhaltsverzeichnis}

$\begin{array}{ll}\text { Abkürzungsverzeichnis } & \text { XIII } \\ \text { Abbildungsverzeichnis } & \text { XVI } \\ \text { Tabellenverzeichnis } & \text { XVII }\end{array}$

1. Kapitel: Problemstellung und Aufbau der Arbeit

1

2. Kapitel: Sozialökonomische Verhaltensforschung 14

$\begin{array}{ll}\text { I. Ziel der sozialökonomischen Verhaltensforschung } & 14\end{array}$

II. Historischer Abriß der sozialökonomischen Verhaltensforschung 18

3. Kapitel: Bisherige Methoden zur Analyse der Aktienkursbildung und deren Grenzen $\quad 25$

I. Fundamentalanalyse $\quad \mathbf{2 5}$

1. Analyse gesamtwirtschaftlicher Faktoren 26

$\begin{array}{ll}\text { 2. Analyse branchenspezifischer Faktoren } & 27\end{array}$

3. Analyse unternehmensindividueller Faktoren $\quad 28$

$\begin{array}{ll}3.1 \text { Substanzwertmethode } & 28\end{array}$

$\begin{array}{ll}3.1 .1 \text { Bilanzwertverfahren } & 28\end{array}$

$\begin{array}{ll}3.1 .2 \text { Liquidationswertverfahren } & 28\end{array}$

$\begin{array}{ll}3.2 \text { Ertragswertmethode } & 30\end{array}$

$\begin{array}{ll}\text { 3.2.1 Dividenden-Discount-Modelle } & 30\end{array}$

$\begin{array}{ll}3.2 .2 \text { Gewinn-Modelle } & 31\end{array}$

3.2.2.1 Steuerbilanzgewinn-Methode 31

3.2.2.2 DVFA/SG-Methode $\quad 32$

4. Grenzen der Fundamentalanalyse 35

II. Technische Analyse $\quad 41$

1. Ursprung der Technischen Analyse 43

1.1 Die Dow-Theorie $\quad 44$

1.2 Die Elliott-Theorie 44

2. Chart-Analyse und computergestützte technische Analyse 45

2.1 Chart-Analyse $\quad 45$

2.2 Computergestützte technische Analyse 51

3. Grenzen der Technischen Analyse 52

III. Random-Walk-Hypothese 53

1. Formulierungen der Random-Walk-Hypothese 55 
1.1 Die strenge Form

$\begin{array}{ll}1.2 \text { Die mittelstrenge Form } & 56\end{array}$

$\begin{array}{ll}1.3 \text { Die schwache Form } & 56\end{array}$

2. Grenzen der Random-Walk-Hypothese $\quad 59$

IV. Fazit

4. Kapitel: Marktteilnehmermorphologie und spezifisches Anlegerverhalten

I. Nicht-professionelle Anleger $\quad 69$

$\begin{array}{ll}\text { 1. Privatanleger } & 69\end{array}$

$\begin{array}{ll}\text { 2. Belegschaftsaktionäre } & 77\end{array}$

3. Großaktionäre $\quad 77$

$\begin{array}{lr}\text { II. Professionelle Anleger } & \mathbf{8 0}\end{array}$

$\begin{array}{lr}\text { 1. Kreditinstitute } & 81\end{array}$

$\begin{array}{ll}1.1 \text { Effektengeschäft } & 81\end{array}$

$\begin{array}{ll}1.2 \text { Anlageberatung } & 84\end{array}$

2. Kapitalanlagegesellschaften $\quad 86$

$\begin{array}{ll}2.1 \text { Analyse und Entscheidungsfindung } & 87\end{array}$

$\begin{array}{ll}2.2 \text { Einengung des Handlungsspielraums } & 89\end{array}$

2.2.1 Marktmäßig determinierte Einengung $\quad 89$

$\begin{array}{lr}\text { 2.2.2 Satzungsmäßig determinierte Einengung } & 89\end{array}$

$\begin{array}{ll}2.2 .2 .1 \text { Anlagezwang } & 89\end{array}$

$\begin{array}{ll}\text { 2.2.2.2 Liquidationszwang } & 90\end{array}$

$\begin{array}{ll}2.2 .3 \text { Benchmarkdenken } & 90\end{array}$

3. Versicherungen $\quad 92$

4. Sonstige Unternehmen 93

$\begin{array}{ll}\text { 5. Kulisse } & 96\end{array}$

$\begin{array}{lr}\text { III. Ausländer } & 97\end{array}$

$\begin{array}{ll}\text { IV. Fazit } & 101\end{array}$

5. Kapitel: Determinanten der Kursbildung 104

$\begin{array}{ll}\text { A. Umwelt des Investors } & 106\end{array}$

I. Faktische Umwelt $\quad \mathbf{1 0 6}$

$\begin{array}{lr}\text { 1. Gesellschaftssystem } & 106\end{array}$

$\begin{array}{lr}1.1 \text { Soziokulturelles System } & 107\end{array}$

$\begin{array}{ll}1.2 \text { Politisches und rechtliches System } & 109\end{array}$

$\begin{array}{lr}1.3 \text { Wirtschaftliches System } & 110\end{array}$ 
2. Markttechnische Determinanten

2.1 Marktstruktur

2.2 Marktsegmente

2.3 Marktbreite

3. Situative Determinanten

3.1 Börsenstimmung

3.2 Aktueller Kontext

II. Soziale Umwelt

1. Unpersönliche soziale Kontakte

1.1 Massenmedien

1.1.1 Printmedien

$\begin{array}{ll}\text { 1.1.2 Elektronische Medien } & 136\end{array}$

$\begin{array}{ll}1.2 \text { Meinungsführer } & 137\end{array}$

2. Persönliche soziale Kontakte $\quad 139$

$\begin{array}{ll}2.1 \text { Bezugsgruppen } & 139\end{array}$

$\begin{array}{ll}2.2 \text { Bezugspersonen } & 141\end{array}$

3. Fazit

I. Informationsaufnahme und -verarbeitung $\quad \mathbf{1 4 6}$

$\begin{array}{ll}\text { 1. Wahrnehmung } & 148\end{array}$

$\begin{array}{ll}1.1 \text { Exkurs Gestaltpsychologie } & 149\end{array}$

1.2 Rationaler Informationsverarbeitungsprozeß 154

1.3 Eingeschränkt rationaler Informationsverarbeitungsprozeß 156

$\begin{array}{ll}\text { 1.3.1 Situative Information } & 161\end{array}$

$\begin{array}{ll}\text { 1.3.1.1 Neuigkeiten } & 162\end{array}$

1.3.1.2 Aktienkurse und Indizes $\quad 163$

$\begin{array}{ll}\text { 1.3.2 Selektive Information } & 164\end{array}$

$\begin{array}{ll}\text { 1.3.2.1 Informationsquelle } & 165\end{array}$

$\begin{array}{ll}\text { 1.3.2.2 Kognitive Dissonanz } & 165\end{array}$

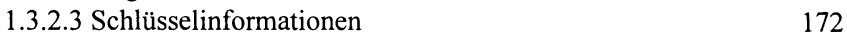

$\begin{array}{ll}\text { 1.3.3 Paradigmatische Information } & 176\end{array}$

$\begin{array}{ll}\text { 1.3.3.1 Schemata } & 176\end{array}$

$\begin{array}{ll}\text { 1.3.3.2 Stereotype } & 181\end{array}$

$\begin{array}{ll}\text { 1.3.3.3 Focal Points } & 184\end{array}$

$\begin{array}{lr}\text { 2. Motivation } & 186\end{array}$

3. Erwartungen 190

3.1. Erwartungsbildung 191

$\begin{array}{ll}\text { 3.1.1 Lernen } & 192\end{array}$

3.1.1.1 Psychologische Lerntheorie $\quad 192$

3.1.1.1.1 Instrumentelle Konditionierung 193

3.1.1.1.2 Klassische Konditionierung 193

3.1.1.2 Information-Processing-Theorie 194

3.1.1.2.1 Lernen durch Wiederholung bzw. 
Einprägung 194

3.1.1.2.2 Lernen durch Einsicht 195

$\begin{array}{ll}\text { 3.1.2 Erfahrung } & 196\end{array}$

3.2 Erwartungsarten und deren Auswirkungen $\quad 198$

$\begin{array}{ll}3.3 \text { Operationalisierung von Erwartungen } & 201\end{array}$

$\begin{array}{ll}3.4 \text { Fazit } & 206\end{array}$

$\begin{array}{lr}\text { II. Informationsbewertung } & \mathbf{2 0 8}\end{array}$

\begin{tabular}{ll} 
1. Heuristiken & 209 \\
\hline 1.1 Reprasentiviatsheuristik & 210
\end{tabular}

$\begin{array}{ll}1.1 \text { Repräsentativitätsheuristik } & 210\end{array}$

$\begin{array}{ll}\text { 1.1.1 Element repräsentativ für die Kategorie } & 211\end{array}$

1.1.2 Stichprobe repräsentativ für die Grundgesamtheit 213

\begin{tabular}{ll}
1.2 Verfügbarkeitsheuristik & 215 \\
\hline
\end{tabular}

1.2.1 Häufigkeit des Auftretens 215

$\begin{array}{ll}\text { 1.2.2 Retrieval Cue } & 216\end{array}$

1.2.3 Augenfälligkeit von Informationen $\quad 216$

$\begin{array}{ll}1.3 \text { Verankerung und Anpassung } & 217\end{array}$

2. Erster Eindruck $\quad 218$

3. Sonstige ausgewählte Biases und Fallacies $\quad 221$

$\begin{array}{ll}3.1 \text { Ambiguität } & 221\end{array}$

3.2 Dispositionseffekt $\quad 225$

$\begin{array}{ll}3.3 \text { Nennwertillusion } & 228\end{array}$

3.4 Sequential Decision-Making Hypothese 229

$\begin{array}{ll}\text { III. Gruppendynamik } & 231\end{array}$

1. Konformitätsdruck 231

2. Risikoschub-Effekt 235

6. Kapitel: Fazit und Ansatz eines interdisziplinären Ansatzes 239

$\begin{array}{ll}\text { Anhang } & 246\end{array}$

$\begin{array}{lr}\text { Literaturverzeichnis } & 260\end{array}$ 


\section{Abkürzungsverzeichnis}

A.: $\quad$ Auflage

Abb.: $\quad$ Abbildung

a.o.: außerordentliche

AG: Aktiengesellschaft

AGB: Allgemeine Geschäftsbedingungen

Art.: Artikel

BAK: Bundesaufsichtsamt für das Kreditwesen

BBankG: Bundesbankgesetz

Bd.: $\quad$ Band

BGB: Bürgerliches Gesetzbuch

BI: Bankinformation

BIP: Bruttoinlandsprodukt

BiRiLiG: Bilanzrichtlinien-Gesetz

BörsG: Börsengesetz

bspw.: beispielsweise

BZ: Börsenzeitung

Calif.: California

CAPM: $\quad$ Capital Asset Pricing Model

DAI: Deutsches Aktieninstitut e.V.

DB: Deutsche Bank

Diss.: Dissertation

DVFA: Deutsche Vereinigung für Finanzanalyse und Anlageberatung

EStG: $\quad$ Einkommensteuergesetz

et al.: et altera

f.: $\quad$ folgende

FAJ: $\quad$ Financial Analysts Journal

FAZ: $\quad$ Frankfurter Allgemeine Zeitung

FED: $\quad$ Federal Reserve Bank

ff.: $\quad$ fortfolgende

Ffm.: $\quad$ Frankfurt am Main

GfK: Gesellschaft für Konsum-, Markt- und Absatzforschung 
$\begin{array}{ll}\text { GG: } & \text { Grundgesetz } \\ \text { ggl.: } & \text { geglättet }\end{array}$

$\mathrm{GmbH}: \quad$ Gesellschaft mit beschränkter Haftung

H.: $\quad \mathrm{Heft}$

HB: Handelsblatt

HGB: Handelsgesetzbuch

Hrsg.: Herausgeber

HV: Hauptversammlung

IAS International Accounting Standards

i.d.R.: $\quad$ in der Regel

i.H.v.: in Höhe von

IR: Investor Relations

i.S.d.: $\quad$ im Sinne der

i.V.m.: $\quad$ in Verbindung mit

i.w.S.: im weiteren Sinne

IBES: Institutional Brokers Estimate System

Ibis: Inter-Banken-Informations-System

Ifo: Institut für Wirtschaftsforschung e.V.

Jg.: Jahrgang

Jo: Journal of

JoASP.: Journal of Abnormal and Social Psychology

JoB: Journal of Business

JoBF: Journal of Banking and Finance

JoF: Journal of Finance

JoFE: Journal of Financial Economics

JoFQA: Journal of Financial and Quantitative Analysis

JoPE: Journal of Political Economy

JoPM: Journal of Portfolio Management

KAGG: Gesetz über Kapitalanlagegesellschaften

KGV: $\quad$ Kurs-Gewinn-Verhältnis

KWG: Gesetz über das Kreditwesen 
N. J.: $\quad$ New Jersey

NY: New York

NYSE: New York Stock Exchange

ÖBA: Österreichisches Bankarchiv

o.J.: ohne Jahresangabe

o.O.: ohne Ortsangabe

OR: Operations Research

o.V.: ohne Verfasser

PER: $\quad$ Price-Earnings-Ratio

Psych. Rev.: Psychological Review

RSI: Relative Strength Index

s.b.: $\quad$ saisonbereinigt

SG: Schmalenbachgesellschaft

Sp.: $\quad$ Spalte

SRC: $\quad$ Survey Research Center

Tab.: Tabelle

US-GAAP United States-General Accepted Accounting Principles

u.U.: $\quad$ unter Umständen

VAG: $\quad$ Versicherungsaufsichtsgesetz

VermBG: $\quad$ Vermögensbildungsgesetz

Vol.: Volume

WW: Wirtschaftswoche

ZEW: Zentrum für Europäische Wirtschaftsforschung

ZfbF: Zeitschrift für betriebswirtschaftliche Forschung

ZfgK: $\quad$ Zeitschrift für das gesamte Kreditwesen

z.T.: $\quad$ zum Teil

z.Z.: $\quad$ zur Zeit 


\section{Abbildungsverzeichnis}

Abb. 1:

Abb. 2:

Abb. 3:

Abb. 4:

Abb. 5

Abb. 6:

Abb. 7:

Abb. 8:

Abb. 9:
Deutsche Bank AG

48

Dyckerhoff AG

79

Relative Stärke Small Caps vs. Blue Chips

125

Dalmatinerhund

151

Rubinscher Becher

Schering AG

169

„Starting Point of a Problem-solving Experiment“

195

Die Kahneman-Tversky „Value Function“

227

ZEW-Finanzmarktreport

259 


\section{Tabellenverzeichnis}

Tab. 1:

Durchschnittskurse deutscher Aktien in

den Wertpapierdepots

Tab. 2:

Aufkaufkandidaten im Überblick

Tab. 3:

International bekannte Standardwerte

99

Tab. 4:

Anteil der $5 \%$ größten Unternehmen an

der gesamten Marktkapitalisierung in \%

Tab. 5

Anteile der $5 \%$ größten Unternehmen an

den gesamten Börsenumsätzen in \%

Tab. 6:

Inländische Aktien mit dem größten Umsatz im Jahre 1995

Tab. 7:

Börsenkaptalisierung in Prozent des BIP/BSP

Tab. 8:

Deutsche Wirtschaftszeitungen

Tab. 9:

Deutsche Wirtschaftsmagazine

Tab. 10:

Informationsquellen professioneller und privater Anleger

Tab. 11:

Beratungsbogen Wertpapiere

Tab. 12:

Standardization Factors and Weights for

Composite Index Components/USA

Tab. 13: 


\section{- XVIII -}

Tab. 14: Investionstätigkeit und Investitionsindikatoren aus

den Ifo-Umfragen

Tab. 15:

Brancheneinteilung des Composite Dax (CDax)

Tab. 16:

Brancheneinteilung des Dax 100

Tab. 17:

IBES Express

Tab. 18:

The Estimate Directory

Tab. 19:

World Equities

256

Tab. 20:

ZEW-Fragebogen

Tab. 21:

ZEW-Finanzmarkttest 